MATTER: International Journal of Science and Technology

ISSN 2454-5880

Low et al., 2022

Volume 7 Issue 3, pp. 69-79

Received: 25 th June 2021

Revised: $7^{\text {th }}$ December 2021, 13 th January 2022

Accepted: 17th January 2022

Date of Publication: 19th January 2022

DOI- $h$ ttps://doi.org/10.20319/mijst.2022.73.6979

This paper can be cited as: Low, K. O., Khoo, K. T., Koon, Y. Y., \& Chua, J. X. (2022). Generation of Electrons in Aqueous Water Using the Principle of Induced Current. Matter: International Journal of Science and Technology, 7 (3), 69-79.

This work is licensed under the Creative Commons Attribution-Non-Commercial 4.0 International License. To view a copy of this license, visit http://creativecommons.org/licenses/by-nc/4.0/ or send a letter to Creative Commons, PO Box 1866, Mountain View, CA 94042, USA.

\title{
GENERATION OF ELECTRONS IN AQUEOUS WATER USING THE PRINCIPLE OF INDUCED CURRENT
}

\author{
Kin Onn Low \\ Director, IOP Specialists Sdn. Bhd., Klang, Malaysia \\ mclidkol1999@gmail.com \\ Kee Tong Khoo \\ Department of Materials Engineering, Tunku Abdul Rahman University College, Faculty of \\ Engineering_and Technology, Kuala Lumpur, Malaysia \\ khookt@tarc.edu.my \\ Ying Yao Koon \\ Director, Million Water Sdn. Bhd, Klang, Malaysia \\ jasonkoon93@gmail.com \\ Jia Xin Chua \\ Chemist, Million Water Sdn. Bhd, Klang, Malaysia \\ chuajx98@gmail.com
}

\begin{abstract}
Drinking water enriched with natural electrons is believed to bring many benefits to people's health because the electrons are able to destroy free radicals in the human body. This work demonstrated that electrons can be generated when aqueous water is forced to flow through a magnetic field created by a magnetic iron oxide source. The electrons created using this method were shown to affine to positively charged particles, which are the hydrogen ions in
\end{abstract}


the aqueous water. Such affinity created hydrogen-electron pairs and consequently increases the concentration of the hydroxyl ions in the water system when the aqueous water is flown through the magnetic field. When this happened, the $\mathrm{pH}$ of the aqueous water increased compared to the aqueous water before flowing through the magnetic field. Similarly, the resistivity of the aqueous water is reduced as a result of the generation of electrons in aqueous water, when the aqueous water flows through the magnetic field.

\section{Keywords}

Aqueous Water, Electrons, Magnetic Fields, Hydrogen-Electron Pairs.

\subsection{Literature Review}

Water is vital for the survival of all lives in the world. Clean, safe, and sufficient freshwater is important to sustain the health of human beings, ecosystem, and smooth functional economies. Hence, numerous researches and studies had been performed to investigate high-quality drinking water with health benefits. Several types of drinking water with improved quality such as reverse osmosis (RO) water (Bhagwat \& Munavalli, 2020), energy water (Smirnov, 2003; Traylor et al., 2011), hydrogen water (Fujita et al., 2009), and alkaline water (Koufman \& Johnston, 2012) have been reported to have numerous health benefits. However, which type of this water is having the best health benefits is still debatable. In addition, there are also limited agreements on which is the best drinking water treatment system.

Many households drinking water is originated from tap water and it is usually boiled or purified using a water filtration or distillation system for consumption. This filtered or distilled water is often undergoing post-treatments to enhance its health benefits, especially with the emerging of energy water which is found to be good for health (Smirnov, 2003; Traylor et al., 2011; Vysotskii et al., 2006; Yamabhai et al., 2014). Energy water in this context is referred to as the normal water that flows through an energy field which can activate the water to possess a higher energy level. This energy-water possesses high liquidity and easily penetrates human body cells (Smirnov, 2003). It contains a higher density of free electrons, increasing the possibility to supply electrons to the human body. Consequently, these electrons donating water acts as an antioxidant or reducing agent to the body which receives electrons. Indeed, as compared to ordinary tap water, this energy-water containing electrons is known to have several health benefits as summarized in the following paragraph (Ignacio et al., 2012; Shirahata et al., 2012; Rahman, 2007; Shigeo, 2011). 
Studies reported that the energy water has distinct viscosity, lower surface tension and smaller molecular structures that can easily penetrate body cells, allowing more hydration and better detoxification in a human body. It is commonly known that an acidic body tends to recruit health issues. Through drinking energy water with a $\mathrm{pH}$ value in the alkaline region, the $\mathrm{pH}$ level of human body fluids can be maintained at 6.9 to 7.2 . This $\mathrm{pH}$ level is effective to energy bacteria, inflammations and diseases from at energy-waterbody. In addition, the ability of the energy water to supply more electrons than ordinary tap water enables the energy water to exhibit higher antioxidant power which can neutralize free radicals. These free radicals, especially oxygen-free radicals can damage the biological system, induce rapid aging and catalyze the development of many fatal diseases including cancer. Studies also show that the antioxidant effect of alkaline water is originated from the highly reactive loosely bounded electrons or free hydroxyl, $\mathrm{OH}^{-}$ions carried in the alkaline water. These electrons or ions are said to have low oxidation-reduction potential (-ORP) which can donate electrons to unstable oxygen radicals and deter the interactions of the active oxygens with normal molecules in the human body.

\subsection{Introduction}

In the view of health benefits of water with high electron density, the authors proposed an effective approach to generate and capture electrons in aqueous water by using the principle of induced current. For the purpose of human drinking, the authors proposed this method because this method of electron addition is not artificially added, rather it is naturally induced by the magnetic field. The authors used magnetic iron oxides to generate magnetic fields due to their ferrimagnetism properties while the water stream acts as kinetic motion. Each particle is magnetic and collectively, they will create an infinite number of magnetic fluxes. As the magnetic flux is disrupted by the flowing aqueous water, electrons are induced. When electrons are generated and captured in the aqueous water during the electrons induction process, a few chemical changes to the aqueous water could potentially benefit the health of people.

\subsection{Research Objectives}

This is a fundamental research work with the purpose to add electrons into the water for drinking and industrial purposes. When electrons are generated, the authors have performed adequate analysis to prove their existence in the aqueous water and to try to find out the subsequent chemical response of the aqueous water towards such additions. Such chemical analysis suggested the formation of hydrogen-electron pairs and the authors expect these reactions should bring more effects than just electron addition. However, this paper will only 
focus on the formation of hydrogen-electron pairs and its finding; other reactions are not discussed.

\subsection{Methodology}

It was important to prepare a suitable magnetic source so that a space consisting of sufficient magnetic fluxes can be produced. When the magnetic space is created, a procedure was assembled so that the aqueous water could be allowed to flow through the magnetic space and could be collected for further analysis. Analysis by various instrumentations was performed.

\subsection{Materials}

In this study, distilled water and tap water from 4 different places are used as the water source. A $600 \mathrm{ml}$ cylindrical container with an internal hollow tube is compactly filled with micronized iron oxide particles. The micronized iron oxide particles are used as received from IOP Specialists Sdn. Bhd., Malaysia. These particles are prepared using a method described in the United States patent (Kin Onn Low, 2008) with the patent number US7347893B2. The powder as described in the patent possessed pigmentation power to many kinds of engineering materials, such power elaborated in the patent has pointed the mean size of the powder to below 1 micrometre.

\subsection{Apparatus: Magnetic Treatment Device}

The apparatus to provide the magnetic treatment to the aqueous water was prepared by feeding magnetite powder (as prepared from Section 2.1.1) into a cylindrical container (base diameter of $6.5 \mathrm{~cm}$ and height of $20 \mathrm{~cm}$ ) with an internal tubular hollow tube of $1 \mathrm{~cm}$ diameter at the centre of the container. The hollow tube was used to accommodate a foreign polyethylene tube with a $6.35 \mathrm{~mm}$ internal diameter as illustrated in Figure 1. This container was constructed using stainless steel SS304.

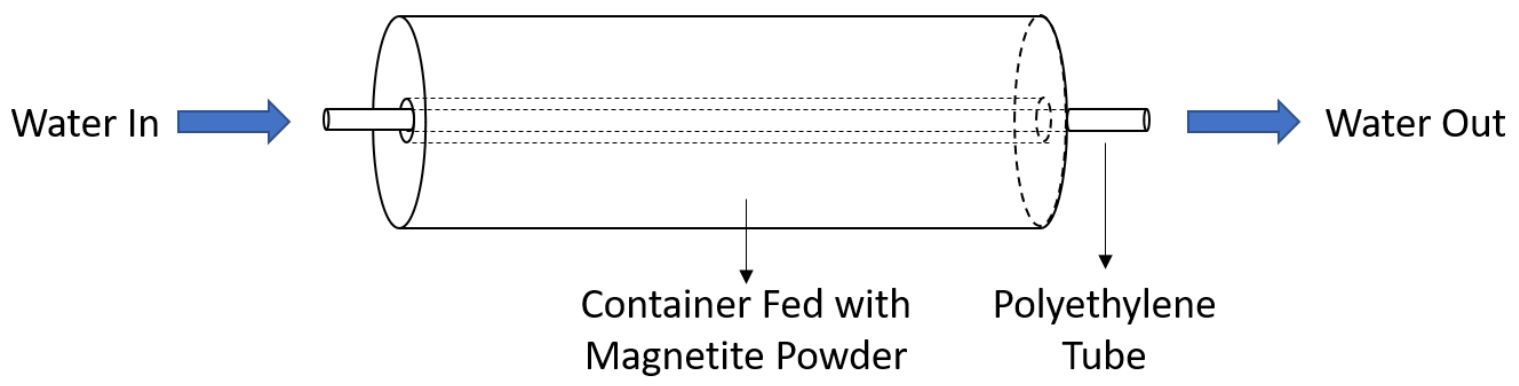

Figure 1: Illustration of water flowing through the container fed with magnetite powder.

(Source: Self) 


\subsection{Apparatus: Water Pumping System}

The water pumping system was employed to provide flow ability to the intended water. This is essential so that the aqueous water can move from the water source to the destination. This movement is crucial to ensure the aqueous water move through the prepared magnetic field.

The water pumping system in this study was made up of 2 polypropylene bottles, 1 peristaltic pump, and polyethylene tubes with diameters of $6.35 \mathrm{~mm}$. The peristaltic pump was used to pump the aqueous water from one bottle (Bottle A) to another bottle (Bottle B) using an interconnected polyethylene tube. All the fittings were tightened to prevent water from leaking during the pumping process. This water pumping system is illustrated in Figure 2.

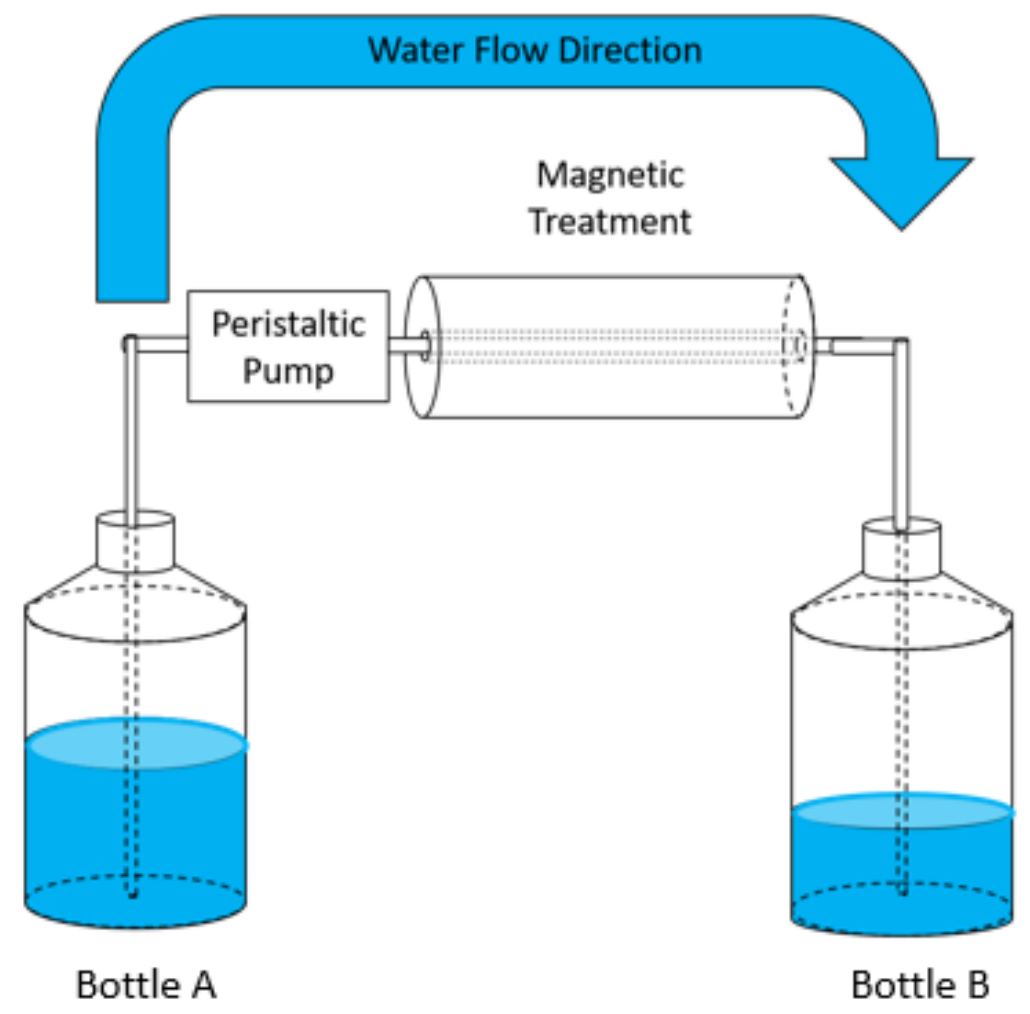

Figure 2: Illustration of the water magnetic treatment (Source: Self)

\subsection{Instrumentation}

The water source as well as all samples collected in Section 2.3 were immediately tested for the $\mathrm{pH}$ and electrical conductivity (EC) using the instrument as listed in Table 1. All measurements were conducted using their standard operating procedures as provided by their respective manufacturers. 
Table 1: Devices for water testing

\begin{tabular}{|c|c|c|}
\hline Property & Device & Manufacturer \\
\hline $\mathrm{pH}$ & $\mathrm{pHep}{ }^{\circledR 4} \mathrm{pH} /$ Temperature Tester - HI98127 & Hanna Instrument \\
\hline EC & Pocket Pro Low Range Conductivity Tester -9531400 & Hach \\
\hline
\end{tabular}

(Source: Devices information.)

\subsection{Experimental Procedure}

In the first experiment, the authors had prepared distilled water collected from a standard evaporator (distillation equipment). A sufficient quantity of distilled water was collected to ensure the full completion of the entire experiment. Distilled water used for the experiment was freshly prepared prior to the experiment. This is to ensure minimum contamination to the distilled water source.

In the second experiment, the authors collected tap water as the source water from four different points randomly. Similarly, tap water used for the experiment was freshly collected prior to the experiment. This is to ensure minimum contamination to the tap water source. The authors applied four tap water sources in four experiments to ensure electrons are actually can be produced in the tap water irrespective of their collection points.

In both experiments, the authors used distilled water and tap water in the experiment because both are aqueous water. Since electron generation uses the principle of the induced current as a widely accepted method, the authors wish to demonstrate electrons can be captured by aqueous water irrespective of the difference in their contents.

When all water sources are prepared, all experiments were carried out as illustrated in Figure 2. Bottle A is the source water and Bottle B is the magnetically treated water; both the source water and the treated water were carried out $\mathrm{pH}$ and conductivity test. All data were collected and presented in the following sections.

\section{Results and Discussion}

The authors have successfully carried out the experiments and were able to collect the resulting water from those experiments. The results obtained were excellent because the authors were able to perform analysis on the data collected. Such analysis showed logical and reasonable future development of this fundamental research work into benefited projects for human kinds. 


\subsection{Electrons Generation in Distilled Water}

The $\mathrm{pH}$ and electrical resistivities of the distilled water before and after flowing through the cylindrical container filled with magnetic iron oxides are tabulated in Table 2.

Table 2: $p H$ values and electrical resistivities of the distilled water before and after flowing through the magnetic fields.

\begin{tabular}{|c|c|c|}
\hline Property & $\begin{array}{c}\text { Water Before Flowing into } \\
\text { Container }\end{array}$ & $\begin{array}{c}\text { Water Leaving the } \\
\text { Container }\end{array}$ \\
\hline $\mathrm{pH}$ & 5.65 & 5.94 \\
\hline Resistivity $/ \mathrm{k} \Omega$ & 185 & 133 \\
\hline
\end{tabular}

(Source: Data collected from experiments.)

According to Table 2, it is found that the distilled water has increased in $\mathrm{pH}$ value but decreased in electrical resistivity after leaving the cylindrical container filled with magnetic iron oxides. This phenomenon can be explained using the principle of induced current. As the water flows through the magnetic field, it cuts the magnetic flux and the electrons are induced within the water. When electrons are generated, this has caused a decrement in the electrical resistivity as indicated in Table 2 . The increment in $\mathrm{pH}$ value implied the increment of the concentration of hydroxyl, $\mathrm{OH}^{-}$ions in water. Both the changes in the $\mathrm{pH}$ values and electrical resistivity of the distilled water can be explained when the authors propose a mechanism for the generation of the electron in water. The proposed mechanism is elaborated further in the following section.

\subsection{Proposed Mechanism for the Electron Induction in Distilled Water}

In a typical case of electrical current induction in a metal conductor, the induced electrical current is due to the conduction of the induced electrons in the metal conductor. However, in this study, the distilled water replaced the metal conductors, used to cut through the magnetic field. Nevertheless, the distilled water shows a significant decline in resistivity after the electrons induction process. Hence, free electrons, $e^{-}$are proposed to be generated in distilled water. Further explanations on the mechanism are elaborated in the following paragraphs.

The water, $\mathrm{H}_{2} \mathrm{O}$ molecules can dissociate into hydrogen, $\mathrm{H}^{+}$cations and hydroxyl, $\mathrm{OH}^{-}$anions as shown in Eq. 1. However, the dissociation is a reversible process and the number of charge carriers and free electrons are low which provide high resistivity in distilled water as shown in Table 2. 


$$
\mathrm{H}_{2} \mathrm{O} \rightleftharpoons \mathrm{H}^{+}+\mathrm{OH}^{-}
$$

In this experiment, when water is flowing through the magnetic field, it is felt likely that free $e^{-}$are generated in the water. These free $e^{-}$will be attracted to the free $H^{+}$cations due to their negative charges to form hydrogen-electron pairs. Such affinity creates metastable or weak bonding between the free $H^{+}$cations and $e^{-}$. Consequently, the equilibrium of the cations and anions in Eq. 1 is disturbed because some of the free $\mathrm{H}^{+}$cations are bonded to those electrons. As a result, the concentration of free $\mathrm{OH}^{-}$anions became higher relative to the concentration of the hydrogen ions; thus, the $\mathrm{pH}$ increases. In addition, these free $\mathrm{OH}^{-}$anions are also charge carriers that can decrease the resistivity of the water as shown in Table 2. A simple illustration to produce water with high electron density is as shown in Figure 2.

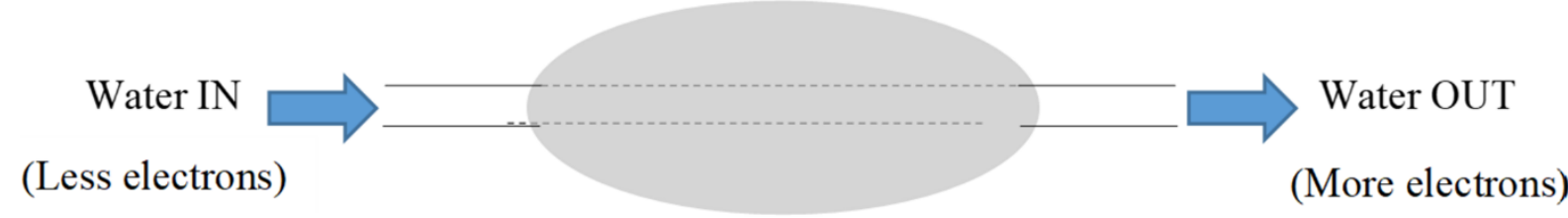

Magnetic field generated by iron oxide particles

Figure 3: Schematic illustration of the proposed electrons generation mechanism in the water flowing through a magnetic field.

(Source: Self)

To estimate the number of hydrogen-electron pairs generated in the water, a calculation is proposed based on the $\mathrm{pH}$ of the water. From Eq. 1, when water is flowing through the magnetic field, the concentration of hydrogen-electron pairs shall be the same number of the free $\mathrm{OH}^{-}$anions. Therefore, by applying Eq. 2, the molarity of hydrogen-electron pairs in the water before and after it flows through the magnetic field can be calculated. The difference between $M_{0}$ and $M_{1}$ is then the molarity of the hydrogen-electron pairs, $M_{2}$, where $M_{0}$ and $M_{1}$ are the molarity of hydroxyl ions before and after leaving the magnetic field.

$$
p H=-\log \left[H^{+}\right]
$$

Subsequently, the number of electrons paired with hydrogen ions, $N_{e^{-}}$in the water due to the electrons induction process can be calculated based on Eq. 3 using the Avogadro constant, $N_{A}$ and if the volume of the water, $V$ is known. The $N_{e^{-}}$is thought to have a linear relationship with the $M_{2}$.

$$
N_{e^{-}}=M_{2} \times V \times N_{A}
$$




\subsection{Electron Induction in Tap Water}

Based on the mechanism proposed in Section 3.2, the proposed mechanism in this study to generate electrons in distilled water shall be applied to all other sources of water. Hence, by this concept, tap water from 4 other random sources are tested using the approach in this study.

The authors had repeated the experiments to 4 kinds of tap water. Table 3 shows the $\mathrm{pH}$ increment of the tap water from 4 different sources after the electrons induction process. As expected, the 4 samples showed an increment in the $\mathrm{pH}$ similar to the distilled water. This has strengthened the findings in Section 3.2.

Table 3: $p H$ increment of tap water from four different sources.

\begin{tabular}{|c|c|}
\hline Tap water & $\mathbf{p H}$ increment of water leaving the container \\
\hline Source 1 & 0.25 \\
\hline Source 2 & 0.53 \\
\hline Source 3 & 0.46 \\
\hline Source 4 & 0.38 \\
\hline
\end{tabular}

(Source: Data collected from experiments.)

However, it is important to note that the extent of $\mathrm{pH}$ increment is different for different sources of tap water and distilled water. It is felt likely that the differences occurred when the tap water contain contaminants or impurities with various concentrations which are not found in the distilled water. However, they exhibited similar results that when electrons are generated, the $\mathrm{pH}$ of the water will be increased due to electron generation.

\section{Conclusion}

In this study, the authors proposed an approach to generate free electrons in the water using the well-known principle of induced current. Initial investigations are conducted on distilled water, further experiments were conducted using tap water, and both kinds of water confirmed the proposed mechanism. All experiments showed that when the water flows through a controlled magnetic field provided by micronized iron oxides particles, resistivity is observed to decline due to the presence of induced electrons. The proposed mechanism elaborated that free electrons were generated during these experiments. The proposed electrons generation mechanism in distilled water took place because there were increments of $\mathrm{pH}$ and decrement of electrical resistivity in the water. In summary, the increment of the $\mathrm{pH}$ value is caused by the generation of electrons in the water. When the electrons are generated, they will adhere to the free hydrogen cation. As a result, the concentration of free hydroxyl anions 
became higher relative to the hydrogen cations because some hydrogen cations have been combined with the generated electrons. Further experiments were conducted using 4 different kinds of tap water to testify the proposed mechanism in this study. Since the $\mathrm{pH}$ of tap water showed a significant increment after the electrons induction process, it is demonstrated that electrons are generated despite the type of tap water. However, the presence of impurities in tap water has caused the increment of $\mathrm{pH}$ to behave differently than that of distilled water. Nevertheless, the generation of the electron in tap water is intact working using the proposed mechanism.

\subsection{Scope of Future Research}

The authors have demonstrated the experiments with the outcomes logical and reasonable to conclude that electrons could be generated in water when water is moving through a space of magnetic fluxes. When electrons are generated, they could be possible chemical changes or reactions that could bring to such additions. It is vital to investigate the reactivity and the benefits that could be accompanied. For example, the authors have observed that when electrons are generated as the result of the hydrogen-electron pair's formation, oxygen could be free off from aqueous water. There should be much more future work than just the oxygen generation when aqueous water receives electrons.

\subsection{Research Limitations}

The authors have foreseen that this fundamental research work may be the fundamental of wide topics of important ecological systems, human lifestyles and industries. All topics required expertise, suitable instrumentations and research methodology from various fields. With limited research funding and facilities in Malaysia, such development may not be easily achieved and it required international cooperation from all parties.

\section{REFERENCES}

Bhagwat D.S., Munavalli G.R. (2020). Global Challenges in Energy and Environment. vol., 109-117.https://doi.org/10.1007/978-981-13-9213-9_11

Fujita K., Seike T., Yutsudo N., Ohno M., Yamada H., Yamaguchi H., Sakumi K., Yamakawa Y., Kido M.A., Takaki A., Katafuchi T., Tanaka Y., Nakabeppu Y., Noda M. (2009). Hydrogen in Drinking Water Reduces Dopaminergic Neuronal Loss in the 1-methyl-4-phenyl-1,2,3,6- tetrahydropyridine Mouse Model of Parkinson's Disease. PLoS One, 4, e7247-e7247https://doi.org/10.1371/journal.pone.0007247 
Ignacio R.M.C., Joo K.-B., Lee K.-J. (2021). Clinical Effect and Mechanism of Alkaline Reduced Water. Journal of Food and Drug Analysis, 20, 394-397. https://doi.org/10.38212/2224-6614.2099

Kin Onn Low, (2008). IRON OXIDE PIGMENTS FROM MILL SCALE, US patent, patent no. US 7,347,893 B2.

Koufman J.A., Johnston N. (2021). Potential Benefits of pH 8.8 Alkaline Drinking Water as an Adjunct in the Treatment of Reflux Disease. Annals of Otology, Rhinology \& Laryngology, 121, 431-434. https://doi.org/10.1177/000348941212100702

Rahman K. (2007). Studies on free radicals, antioxidants, and co-factors. Clinical Interventions in Aging, 2, 219-236. PMID: 18044138

Shigeo O. (2011). Recent Progress Toward Hydrogen Medicine: Potential of Molecular Hydrogen for Preventive and Therapeutic Applications. Current Pharmaceutical Design, 17, 2241-2252.https://doi.org/10.2174/138161211797052664

Shirahata S., Hamasaki T., Teruya K. (2012). Advanced research on the health benefit of reduced water. Trends in Food Science \& Technology, 23, 124-131. https://doi.org/10.1016/j.tifs.2011.10.009

Smirnov I. (2003). Activated Water. Electronic Journal of Biotechnology, 6, 128-142.

Retrieved from http://www.bioline.org.br/request?ej03016

Traylor M.J., Pavlovich M.J., Karim S., Hait P., Sakiyama Y., Clark D.S., Graves D.B. (2011). Long-term antibacterial efficacy of air plasma-activated water. Journal of Physics D: Applied Physics, 44, 472001. https://doi.org/10.1088/0022$\underline{3727 / 44 / 47 / 472001}$

Vysotskii V.I., Olishevsky S., Yanish Y.V., Kornilova A.A. (2007). Investigation of Physical Properties of MRET Activated Water and its Successful Application for Prophylaxis and Treatment of Oncology. World Congress on Medical Physics and Biomedical Engineering 2006, 14, 1902-1905. https://doi.org/10.1007/978-3-540-36841-0_474

Yamabhai M., Chumseng S., Yoohat K., Srila W. (2014). Diverse biological effects of electromagnetic-treated water. Homeopathy: The Journal of the Faculty of Homeopathy, 103, 186-192. https://doi.org/10.1016/j.homp.2013.11.004 